\title{
Variaciones en el contenido de energía en músculos de Salminus brasiliensis en diferentes estados de desenvolvimiento gonadal en el área de influencia del reservatório de Manso, Mato Grosso, Brasil
}

\author{
Luis A. Espínola ${ }^{1}$, Wladimir M. Domingues ${ }^{2} \&$ Evanilde Benedito $^{3}$
}

1. Programa de Pós-graduacão em Ecologia de Ambientes Aquáticos Continentais-Universidade Estadual de Maringá, Avenida Colombo, 5790, 87020-900 Maringá, Paraná. (luisespo00@ hotmail.com)

2. Núcleo de Pesquisas em Limnologia, Ictiologia e Aqüicultura (Nupélia) - Universidade Estadual de Maringá, Avenida Colombo, 5790, 87020-900 Maringá, Paraná. (domingueswm@ @upelia.uem.br)

3. Laboratório de Ecologia Energética, Núcleo de Pesquisas em Limnologia, Ictiologia e Aqüicultura (Nupélia)/Programa de Pósgraduacão en Ecologia de Ambientes Aquáticos Continentais (PEA) - Universidade Estadual de Maringá, Avenida Colombo, 5790, 87020-900 Maringá, PR. (eva@ nupelia.uem.br)

\begin{abstract}
Variations in the content of energy in muscle of Salminus brasiliensis in differents states of the gonadal development in the area of influence of the Manso reservoir Mato Grosso. In order to achieve effectiveness in conservation and management measures it is necessary to understand the origin and destiny of energy by which organisms compete in different environments. This study tested the hypothesis that there are differences in muscle energy content during the gonadal development of Salminus brasiliensis (Cuvier, 1816) in Manso Reservoir. Fish collections were carried out from October 2002 to September 2003. After biometry, muscle samples of all individuals were dried at $60^{\circ} \mathrm{C}$ and dry weight calorie/gram was determined by acalorimetric pump. Condition factor and Gonad Somatic Relationship (RGS) were compared to caloric values. Significant differences were not detected for caloric values between males and females. However, caloric values of each sex were significantly different among stages of gonadal development. There are different tendencies between sexes for ripening individuals: energy content increases in females, while it remains the same in males. For both sexes, higher levels of energy occurred at the mature stage in relation to the other stages. Females presented better condition factor than males, with higher values for mature females and spent males. Females presented higher RGS than males in ripening and mature stages. Intraspecific differences in muscle energy content suggest that similar studies should take all stages of gonadal development into account for both sexes. Abiotic conditions of Brazilian water bodies can also conduct to alterations in fish issue energy concentrations. Studies of energy content should contribute especially to assess anthropogenic impact in aquatic environments, as well rational measures of resource exploitation.
\end{abstract}

KEYWORDS. Energetic content, Gonadal maturation, Salminus brasiliensis, Manso reservoir.

RESUMEN. Para que las medidas de conservación y manejo sean efectivas es necesario el entendimiento del origen y destino de la energía por las cuales compiten los organismos en los diferentes ambientes. Con la hipótesis de que existen diferencias en la energía contenida en los músculos durante el desenvolvimiento gonadal de Salminus brasiliensis (Cuvier, 1816), fueron realizadas colectas entre octubre de 2002 a septiembre de 2003, en el reservatório del río Manso. Después de la obtención de los datos biométricos, de cada individuo se tomaron muestras de la musculatura, las cuales fueron secadas a $60^{\circ} \mathrm{C}$ y el contenido calórico (cal/g de peso seco) determinado en una bomba calorimétrica. Los valores del factor de condición y de la Relación Gónada Somática (RGS) fueron comparados con los contenidos calóricos. No fueron identificadas diferencias significativas entre los valores calóricos de machos y hembras de S. brasiliensis. Sin embargo, se pudo constatar diferencias calóricas significativas en los diferentes estados de maduración gonadal tanto en machos como en hembras. Se observaron tendencias diferentes entre los sexos cuando las gónadas de los individuos se encontraban en maduración: para las hembras, la energía aumentó, mientras que para los machos ésta se mantuvo, indicando una mayor variación energética relacionada mas con la maduración gonadal que con el proceso de migración. En ambos sexos, el estado maduro mostró mayor contenido energético en relación con los demás estados. Los valores del factor de condición, para las hembras, fueron mayores que para los machos, siendo que los mayores valores fueron constatados para hembras maduras y para machos esgotados. La RGS fue más elevado para las hembras, que para los machos en los estados maduración y maduro. Se concluye que debido a las variaciones intraespecíficas asociadas a la maduración gonadal, es importante que se tenga información sobre todos los estados del desenvolvimiento gonadal, para ambos sexos. Las condiciones abióticas en que se encuentran los principales cuerpos de agua brasileños también pueden conducir a alteraciones en las concentraciones de energía presentes en los tejidos de los peces. Estudios sobre el uso de la energía pueden ser útiles a las evaluaciones de impacto, sobre todo en lo que respecta a las interferencias antrópicas en el medio acuático, así como a las medidas de administración racional de los recursos a ser explotados.

PALABRAS CLAVES. Contenido energético, Maduración gonadal, Salminus brasiliensis, Reservatório de Manso.

Los organismos vivos disponen de recursos energéticos finitos a ser canalizados en las actividades de crecimiento, manutención y reproducción. El conocimiento de la manera en que cada organismo distribuye la energía obtenida del alimento consumido en las distintas demandas vitales, es de interés fundamental dado que esta distribución muchas veces puede representar un punto de conflicto para el organismo en determinadas etapas de su vida (PIANKA, 1982). Este interés produjo un incremento en las últimas décadas del uso de modelos bioenergéticos, testando principalmente densidades calóricas del predador y sus posibles presas (BRYAN et al., 1996).

Sin embargo, estudios de bioenergética sobre la ictiofauna tropical son raros (NGAN et al., 1993). En Brasil, este tipo de investigación está restricto a peces marinos y en agua dulce la calorimetría ha sido limitada para las especies de la cuenca del alto río Paraná (Doria \& Andrian, 1997; Benedito-Cecilio \& Morimoto, 2002; VisMARA et al., 2004; DouRADO et al., 2005). 
La construcción de reservatórios durante estos últimos cincuenta años, sobre todo en el alto río Paraná, impuso una serie de restricciones en el ciclo biológico normal de los peces, relacionadas principalmente con la interrupción de las vías migratorias, mitigación del hábitat y cambios en la calidad del agua (PETRERE JR., 1996).

Una de las especies que más ha sufrido con estos impactos es el dorado, Salminus brasiliensis (Cuvier, 1816), siendo una especie que presenta extensas migraciones reproductivas, el acumulo de lípidos y el consecuente incremento calórico es de gran importancia para su crecimiento y reproducción. Estos dos procesos varían en las diferentes fases del desenvolvimiento, actuando de varios modos en los individuos de cada sexo de la misma especie y también entre las diferentes especies.

Salminus brasiliensis es una de las especies más apreciadas para la pesca profesional y deportiva (LowEMCCONNEL, 1975; BANDUCCI JR., 2000; BARBIERI et al., 2001). En cambio, para la piscicultura, presenta alto índice de canibalismo (COMPANHIA ENERGÉTICA DE MinAs GERAIS, 2000), en los primeros días de vida. Sin embargo, está entre las 50 especies de peces mas cultivadas, experimentalmente, para introducción (VIEIRA \& POMPEU, 2001) o comercialmente (Ostrensky, 2000). Como consecuencia de una serie de factores socioeconómicos, políticos, culturales y ecológicos, se aprecia una decadencia en los niveles poblacionales de esta especie, revelada por el mayor esfuerzo de captura de los pescadores comerciales.

La determinación del contenido de energía en los tejidos de animales constituye una herramienta esencial para conocer la estructura y el funcionamiento del flujo de energía en los ecosistemas acuáticos. De esta forma, para que las medidas de conservación y manejo sean efectivas es necesario el entendimiento del origen y destino de los recursos por los cuales compiten los organismos en los diferentes ambientes (SVERLIJ \& EsPinach Ros, 1986). Por este motivo la hipótesis del presente estudio es que existen diferencias en la distribución de energía durante la maduración gonadal, siendo el objetivo del trabajo aportar datos al conocimiento general de la biología de $S$. brasiliensis en un ambiente recién formado, el reservatório de río Manso.

\section{MATERIAL Y MÉTODOS}

El río Manso nace en la Sierra Azul y recorre un valle sinuoso y encajado, recibiendo otros tributarios en ambas márgenes. El reservatório de río Manso se localiza en el estado de Mato Grosso (14 $32^{\circ}$ ' $-15^{\circ} 40^{\prime} \mathrm{S}, 5^{\circ} 40^{\circ}$ '$\left.55^{\circ} 55^{\circ} \mathrm{O}\right)$, próximo al Parque Nacional Chapada dos Guimarães (Fig. 1). El río Manso donde fue construido dicho reservatório, es el principal afluente del río Cuiabá (De Filippo \& SoAres, 1996).

Las colectas de $S$. brasiliensis, fueron realizadas entre octubre de 2002 a septiembre de 2003, de 8 en 8 horas, utilizándose para la captura de la especie: redes, espíneles y tarrafas. Exemplares de la especie fueron depositados en la colección de ictiologia del Núcleo de Pesquisas en Limnologia, Ictiología e Aqüicultura (NUPELIA) en la Universidad Estadual de Maringá.

Los datos biométricos como longitud total (Lt, $\mathrm{cm}$ ), estándar ( $\mathrm{Ls}, \mathrm{cm})$, peso total (Wt, g) y los biológicos como sexo, estadio de maduración gonadal y peso de la gónada $(\mathrm{Wg}, \mathrm{g})$, fueron tomados in situ mediante una balanza analítica con precisión de $0,001 \mathrm{~g}$ y conservadas en alcohol 70\%. El desenvolvimiento de las gónadas fue determinado macroscópicamente, considerándose las características relacionadas al color, transparencia, vascularización superficial, flacidez, tamaño y posición en la cavidad abdominal. Específicamente para saber el desenvolvimiento de los ovarios, fue observado el grado de visualización de los ovocitos. Para determinar dicho desenvolvimiento, se utilizó la escala de maduración de VAZZOLER (1996), constituida por los siguientes estados: inmaduro (imt), reposo (rep), maduración (mat), maduro (mad) y esgotado (esg).

En cada individuo se tomaron muestras de la musculatura de la base de la aleta dorsal. Las muestras fueron conservadas en hielo y trasportadas al laboratorio de Ecología Energética de NUPÉLIA en la Universidad Estadual de Maringá, donde fueron secadas en estufa a $60^{\circ} \mathrm{C}$, hasta peso constante. Enseguida, fueron maceradas en molino de esferas hasta la obtención de polvo fino. El peso de la muestra resultante, la cual varió entre 0,6 a $0,9 \mathrm{~g}$ fue establecido a través de una balanza analítica con precisión de $0,001 \mathrm{~g}$. Para determinar el contenido calórico (caloría/gramo de peso seco), fue utilizada una bomba calorimétrica modelo PARR 1261.

El cálculo del factor de condición (K), fue realizado a través de la expresión: $\mathrm{K}=\left(\mathrm{Wt} / \mathrm{Ls}^{\mathrm{b}}\right) * 1000$ (VAZZOLER, 1996) y el parámetro "b" fue obtenido a través de la regresión entre la longitud estándar y el peso total para ambos sexos. Para la determinación del efecto de la maduración gonadal sobre el peso del cuerpo, se calculó la Relación Gónada Somática (RGS), siendo RGS=(Wg/ Wt)*100 (VAZZOLER, 1996).

Después de la inspección gráfica de las medias y desvíos estándar de los valores calóricos y del factor de condición, se utilizó ANOVA para determinar diferencias

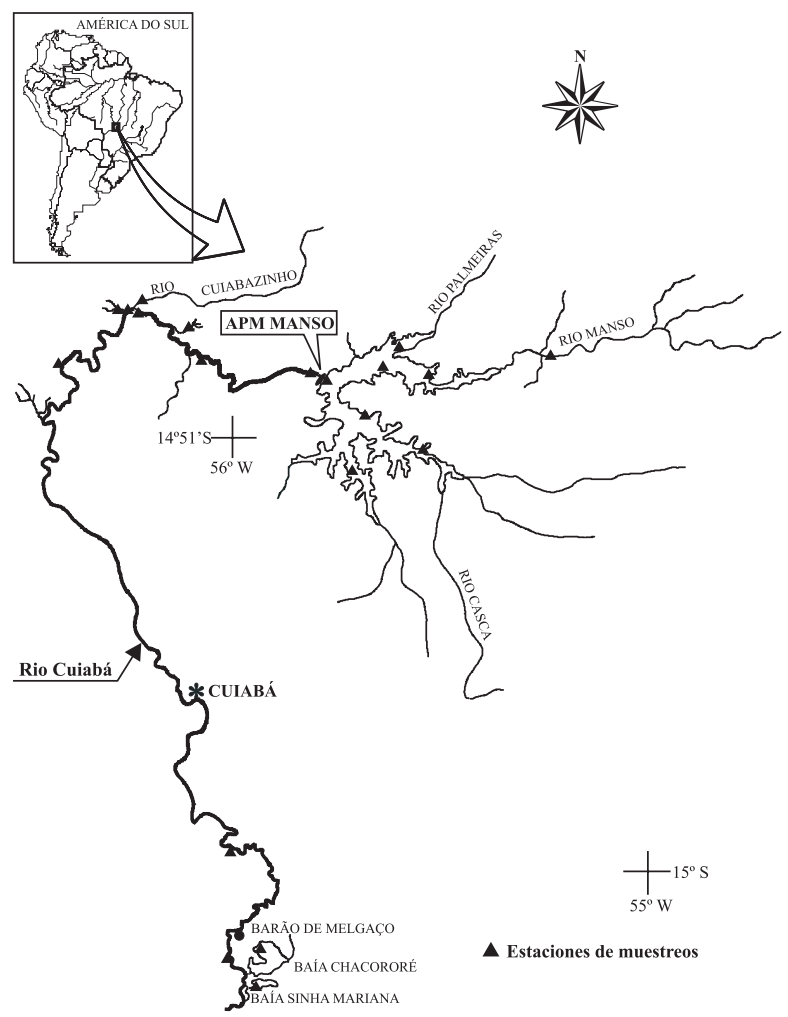

Fig. 1. Área de estudio, localización de las estaciones de muestreos en el reservatório de Manso, río Cuiabá y cuenca del río Paraguay, en el Estado de Mato Grosso. 
significativas entre ellas, considerándose cada sexo por separado (unifactorial) o ambos (bifactorial), las cuales fueron identificadas a través del test a posteriori de Tuckey. Para todos los análisis estadísticos se utilizó el software estadístico STATISTICA 6.0 (STATSOFT, 2003).

\section{RESULTADOS}

No se identificaron diferencias significativas entre los valores calóricos de machos $(\mathrm{n}=191)$ y hembras $(\mathrm{n}=129)$ de . . brasiliensis $(\mathrm{F}=1,88 ; \mathrm{p}=0,173)$ (Fig. 2). Tanto las medias como los desvíos fueron semejantes.

Sin embargo, cuando se compararon las calorías de cada sexo en los diferentes estados de maduración, se constataron diferencias significativas $(F=6,53 ; p<0,05)$. Los machos en los estados imt y rep, son más calóricos que las hembras (Fig. 3). Se observaron tendencias diferentes entre los sexos cuando los individuos se encuentran en el estadio mat: para las hembras, la energía

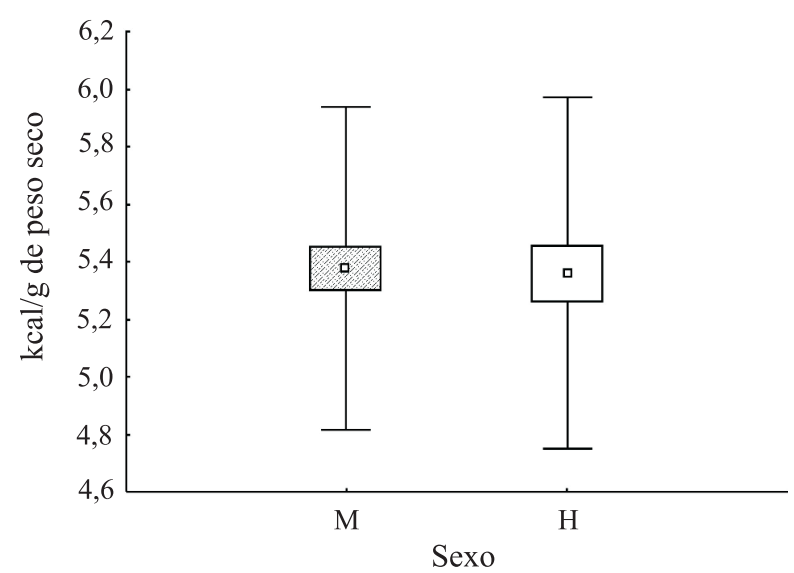

Fig. 2. Valores calóricos medios (cuadrados menores), error (cuadrados mayores) y desvío estándar (líneas verticales) para machos (M) y hembras (H) de Salminus brasiliensis (Cuvier, 1816), colectados en las inmediaciones del reservatório de Manso (MT), entre octubre de 2002 a septiembre de 2003.

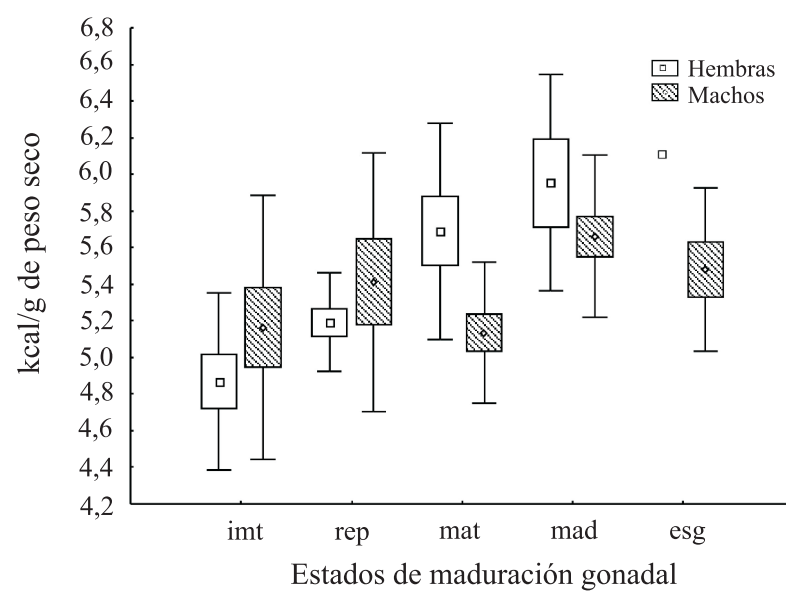

Fig. 3. Valores calóricos medios (cuadrados menores), error (cuadrados mayores) y desvío estándar (líneas verticales) para los estados de maduración gonadal de machos y hembras de Salminus brasiliensis (Cuvier, 1816), colectados en las inmediaciones del reservatório de Manso (MT), entre octubre de 2002 a septiembre de 2003 (imt, imaturo; rep, reposo; mat, maduración; mad, maduro; esg, esgotado). aumenta, mientras que para los machos esta disminuye. En ambos sexos el estadio mad es el más rico en energía, en relación a los demás. En los machos, en el estado esg, fue posible verificar una disminución en la energía, mientras que en las hembras no fue posible ninguna constatación pues solamente fue muestreado un individuo con valor calórico superior al del estado mad.

Los parámetros de la regresión peso total-longitud estándar fueron para las hembras: $a=0,0103 ; b=3,1955$; $r=0,9959$ y para los machos: $a=0,0103 ; b=3,1942 ; r=0,9959$.

El análisis del factor de condición (K) de machos y hembras para los estados de maduración gonadal verifico que, el K para las hembras, fue mas elevado, mostrando una mejor condición que los machos, siendo que los mayores valores fueron constatados para hembras mad y para machos esg (Fig. 4). Las mayores variaciones fueron registradas en el estadio rep para los machos. Los valores de RGS fueron más elevados para las hembras, que para los machos en los estados mat y mad (Fig. 5).

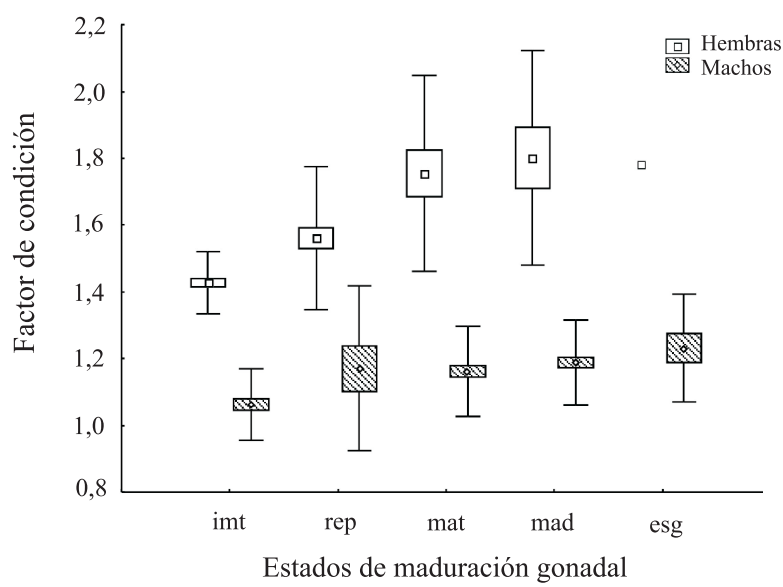

Fig. 4. Valores medios (cuadrados menores) del factor de condición, error (cuadrados mayores) y desvío estándar (líneas verticales) para los estados de maduración gonadal de machos y hembras de Salminus brasiliensis (Cuvier, 1816), colectados en las inmediaciones del reservatorio de Manso (MT), entre octubre de 2002 a septiembre de 2003 (imt, imaturo; rep, reposo; mat, maduración; mad, maduro; esg, esgotado).

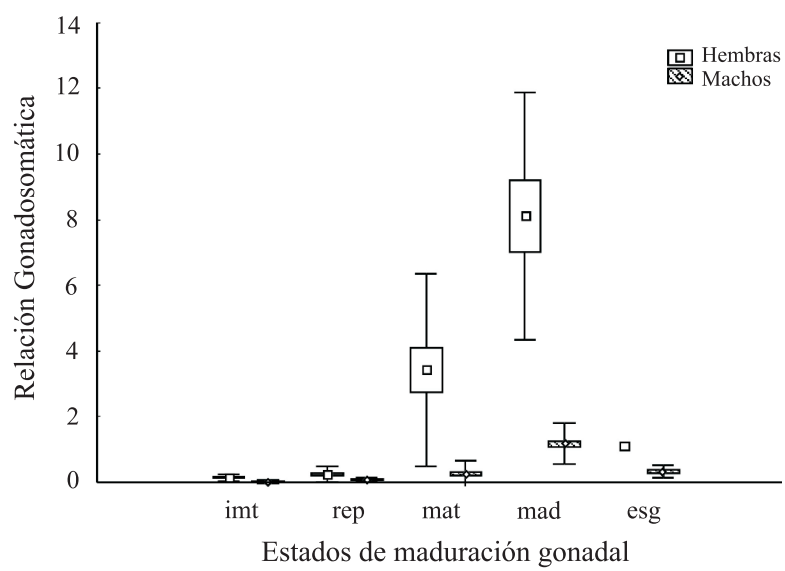

Fig. 5. Valores medios (cuadrados menores) de la Relación Gonadosomática, error (cuadrados mayores) y desvío estándar (líneas verticales) para los estados de maduración gonadal de machos y hembras de Salminus brasiliensis (Cuvier, 1816), colectados en las inmediaciones del reservatorio de Manso (MT), entre octubre de 2002 a septiembre de 2003 (imt, imaturo; rep, reposo; mat, maduración; mad, maduro; esg, esgotado). 


\section{DISCUSSIÓN}

La ausencia de diferencia significativa, que se encontraron en los individuos de Salminus brasiliensis cuando se analizaron los sexos independientes de los estados de maduración gonadal y la presencia de diferencia significativa, cuando los estados fueron considerados, indica que en los estudios ecológicos el predominio en el número de muestras de uno de los estados puede conducir a conclusiones erróneas. Así, existen diferencias entre machos y hembras, dependiendo de la fase en que se encuentra la maduración gonadal.

Salminus brasiliensis es considerada una especie esencialmente piscívora (AlmeIDA et al., 1997; THÉ \& Nordi, 2000; Esteves \& Pinto-Lôbo, 2001). Hay que destacar que el valor calórico medio registrado para esta especie fue superior al observado para otras especies pertenecientes al mismo nivel trófico como Serrasalmus marginatus Valenciennes, 1836 estudiado por VISMARA et al. (2004) y diez otros piscívoros estudiados en cinco reservatórios paranaenses por DouRADo et al. (2005). Los contenidos calóricos de un pez perteneciente a un determinado nivel trófico varían dependiendo del ambiente y de las alteraciones a las que esta sometido. Benedito-CECilio et al. (2005) constataron que los indicadores fisiológicos de Leporinus friderici (Block, 1974), se alteraron cuando el ambiente también sufre cambios estructurales, de río para reservatório. En el reservatório de Manso no fue posible determinar el efecto de esta transformación, considerándose que no están disponibles los resultados previos al represamiento.

Alteraciones calóricas en los músculos de machos y hembras durante la maduración gonadal indican que hay un efecto en la reproducción sobre la cantidad de energía. Salminus brasiliensis es un desovador total que presenta estaciones de desove bien definidas (VAZOLLER, 1996). Los mayores valores tanto para la caloría como para el factor de condición fueron registrados en los músculos de las hembras para los estados mat y mad. BARBIERI et al. (2001) identificaron que los mayores valores del Índice Hepatosomático y del Viscerosomático de $S$. brasiliensis fueron también obtenidos en el período prédesove, el cual fue reduciéndose durante el desove. La reducción hepática esta relacionada a la vitelogénesis, en tanto que la lipídica está relacionada a la migración y al desove. Para el presente estudio, se verificó que solamente las hembras presentaran variaciones calóricas en el desarrollo gonadal, así se supone que el contenido de energía de la especie esté mas relacionado con la maduración gonadal que con la migración para desovar.

Por ser una especie que migra para reproducirse, las hembras evidencian un mayor gasto energético que los machos para los mismos estados. Esto se debe porque ocurre una transferencia de las reservas acumuladas en las vísceras, musculatura o hígado para suplir el desenvolvimiento gonadal, culminando con el desove (Godoy, 1975). Es notoria la variación del peso de las gónadas con relación al peso del cuerpo (RGS) de los individuos en los diferentes estados de maduración gonadal. Lima et al. (1986) estudiando esta especie de la misma forma, constataron variaciones abruptas en el peso de las gónadas de las hembras con relación a la de los machos. Los valores de RGS, del presente estudio confirman esta constatación. En las fases próximas a la reproducción se verifica un aumento del volumen y peso de los ovarios y testículos, siendo mayor para las hembras. Estas además de poseer una alta fecundidad, un ovario en su plenitud, pesa alrededor de $1 \mathrm{~kg}$, el cual representa poco mas del $10 \%$ del peso del cuerpo, conteniendo de 152.900-2.169.000 óvulos (VAZZOLER \& MENEZES, 1992).

En cambio, para los machos de $S$. brasiliensis, el peso de las gónadas maduras representa 1,9\% del peso total de las hembras, siendo que el RGS alcanza 12,08 (Lowe-McConnel, 1999). En el presente estudio, las hembras de la especie en estudio presentaron, un RGS cinco veces mayor que el de los machos. Esta proporción también fue observada por BARBIERI et al. (2001) en el río Mogi-Guaçu (cuenca del río Paraná). Estos autores constataron, que el período en que se registraron indivíduos mat y mad, fue aquel en el que los estómagos estaban repletos de alimento. Estos resultados también fueron confirmados por Esteves \& Pinto-LôBo (2001). Conforme Lima et al. (1986), las hembras invierten más que los machos al ingerir alimento durante el período de reproducción.

Sin embargo, se pudo observar que el estadio rep de los machos presenta mayor media calórica que el de las hembras. Esto indica que los machos inician el nuevo ciclo reproductivo con más energía en los músculos que las hembras.

En este estudio, la falta de hembras de $S$. brasiliensis en el estado esgotado fue motivado por el carácter migratorio de la especie (VAzOLLER, 1989). Posiblemente, el desove puede ocurrir en ambientes distintos al considerado en este estudio.

Los valores medios de RGS y del K distribuidos por los estados de maduración gonadal se correlacionaron con los valores calóricos. Entre las especies estudiadas por VISMARA et al. (2004), solamente las hembras maduras de Loricariichthys platymetopon Isbrücker \& Nijssen, 1979, tuvieron los mayores valores de K. Entretanto, no hay una correlación próxima entre estas dos especies, tanto ecológica (dieta y estrategias reproductivas) como taxonómicas.

HeRbinger \& Friars (1991) observaron para el salmón, Salmo salar (Linnaeus, 1758) una fuerte y significativa correlación entre el factor de condición y el contenido total de lípidos. Sin embargo, los autores excluyeron a los machos maduros del análisis por asumir que estos individuos presentan bajo contenido de gordura en los músculos y en las vísceras siendo que el elevado valor de $\mathrm{K}$ estaba asociado al tamaño de los testículos y no la presencia de grandes cantidades de reservas. En este caso, la variación del factor de condición está relacionada con la variación de la gordura mesentérica y no con la gordura muscular. VISMARA et al. (2004) encontraron para tres especies de la planicie de inundación del alto Paraná que los estados mat y mad presentaron los mayores valores en el factor de condición así como en los de calorimetría.

Así, por haber diferencias intra-específicas en el contenido de energía, se recomienda a los investigadores que tengan en cuenta todos los estados del 
desenvolvimiento gonadal para ambos sexos. La energía de $S$. brasiliensis también puede ser afectada por factores inter-específicos, como el parasitismo (RoDRIGUES et al., 2002) mismo que por condiciones fisiológicas inherentes a cada uno de los sexos, como fue observado por RANZANIPAiva et al. (2003) cuando identificaron el efecto del desenvolvimiento gonadal en el porcentaje de leucocitos en la sangre periférica de las hembras de esta misma especie.

Además de los factores bióticos, las condiciones abióticas en que se encuentran los principales cuerpos de agua brasileños también pueden conducir a alteraciones en las concentraciones de energía presente en los tejidos de los peces. Fuentes de polución, como industrias de alcohol, azúcar, papel y celulosa, así como los residuos domésticos han afectado las principales poblaciones de peces en los últimos años (EsTEVES \& Pinto-Lôbo 2001). De este modo, estudios sobre el uso de la energía deben ser incentivados y sus resultados deben servir de subsidios indispensables en las evaluaciones de impacto, sobre todo cuando se trata de interferencias antrópicas en el medio acuático, así como de las medidas de administración racional de los recursos a ser explotados.

Agradecimientos. A Nupélia, por el apoyo en las colecta de datos, en especial a la Bióloga Marli Cristina Campos; a FURNAS Centrales Eléctricas por el apoyo logístico y al Dr. Luiz Carlos Gomes por el auxílio en la revisión del abstract.

\section{REFERENCIAS BIBLIOGRÁFICAS}

Almeida, V. L. L.; Hahn, N. S. \& Vazzoler, A. E. A. M. 1997. Feeding patterns in five of the high Paraná River floodplain (PR, Brazil). Ecology of Freshwater Fish 6:123-133.

BANDucCI JR., A. 2000. Hidrovia Paraguai-Paraná: ameaça às comunidades pantaneiras. Ciência Hoje 159:62-66.

Barbieri, G.; Salles, F. A. \& Cestarolli, M. A. 2001. Reproductive and nutritional dynamics of Salminus maxillosus Valenciennes, 1849 (Pisces, Characidade) at Mogi-Guaçu river, state of São Paulo, Brazil. Acta Scientiarum 2:441-444.

Benedito-Cecilio, E. \& Morimoto, M. 2002. Effect of preservatives on caloric density in the muscles of Hoplias aff. malabaricus (Bloch, 1794) (Osteichthyes, Erythrinidae). Acta Scientiarum 2:489-492.

Benedito-Cecilio, E.; Pereira, A. L.; Faria, A. C. A. \& Baleroni, H. 2005. Effects of habitat on physiological indicators in Leporinus friderici (Pisces, Anostomidae) in the influence area of the Corumbá reservoir, Goiás, Brazil. Acta Limnologica Brasiliensia 17(1):71-79.

Bryan, S. D.; Soupir, C. A.; Duffy, W. G.; Freiburger, C. E. 1996. Caloric densities of three predatory fishes and their prey in Lake Oahe, South Dakota. Journal of Freshwater Ecology 11(2): 153-161

Companhia Energética de Minas Gerais. 2000. Guia ilustrado de Peixes da bacia do rio Grande. Belo Horizonte, CEMIG. 141p.

De Filippo, R. \& Soares, C. B. P. 1996. Relatório da fase rio. Rio de Janeiro: Furnas Centrais Elétricas. 21p

Doria, C. R. C. \& ANDrian, I. F. 1997. Variation in energy content of somatic and reproductive, tissues related to the reproductive cycle and feeding of female Pimelodus maculatus Lacépède, 1803 (Siluriformes, Pimelodidae) and Schizodon borellii Boulenger, 1895 (Characiformes, Anostomidae). Revista Unimar 2:421-437.
Dourado, E. C. S.; Benedito-Cecilio, E. \& Latini, J. D. 2005. O grau de trofia do ambiente influencia a quantidade de energia dos peixes? In: Rodrigues, L.; Thomaz, S. M.; Agostinho, A. A. \& Gomes, L. C. eds. Biocenoses em reservatórios. padrões espaciais e temporais. São Carlos, Rima. p.211-222.

Esteves, K. E. \& Pinto-Lôbo, A. V. 2001. Feeding pattern of Salminus maxillosus (Pisces Characidae) at Cachoeira das Emas, Mogi-Guaçu river (São Paulo State, Southeast Brazil). Revista Brasileira de Biologia 2:267-276

Godoy, M. P. 1975. Peixes do Brasil: suborden Characoidei. Bacia do rio Mogi Guassu. Piracicaba, Franciscana. v.2. $178 \mathrm{p}$.

Herbinger, C. M. \& Friars, G. W. 1991. Correlation between condition factor and total lipid content in Atlantic salmon, Salmo salar L., parr. Aquaculture and Fisheries Management 22:527-529.

Lima, R. L. V.; Val-sella, M. V. \& Vasco Campos Torquato, L. 1986. Organização e desenvolvimento das gônadas do dourado (Salminus maxillosus Val.) durante o ciclo reprodutivo. Boletim de Fisiologia Animal 10:139-154.

Lowe-McConnell, R. H. 1975. Fish communities in tropical freshwaters: their distribution, ecology and evolution. London, Longman. 337p.

1999. Estudos ecológicos de comunidades de peixes tropicais. São Paulo, EDUSP. 535p.

NGan, P. V.; Gomes, V.; Morais, D. M. \& Passos, M. J. A. C. R. 1993 Estudo bioenergético de animais marinhos costeiros. Instituto Oceanográfico 10:199-215

Ostrensky, A. O. 2000. Milagre da multiplicação dos peixes. Folhas técnicas em Aqüicultura. Série Opinião 1:1-7.

Petrere JR., M. 1996. Fisheries in large tropical reservoirs in South America. Lakes \& Reservoirs: Reservoirs Management 2:111-133.

PianKa, E. R. 1982. Ecología evolutiva. Barcelona, Omega. 363p.

Ranzani-Paiva, M. J. T.; Rodrigues, E. L.; Veiga, M. L.; Eiras, A. C. \& CAmpos, B. E. S. 2003. Contagem diferencial de leucócitos em dourado, Salminus maxillosus Valenciennes, 1840, no rio MogiGuaçu, Pirassununga, SP. Brazilian Journal of Biology 3:517-525.

Rodrigues, E. L.; Ranzani-Paiva, M. J. T. \& Santos, A. A. 2002. Alterações histopatológicas em fígado de dourado Salminus maxillosus Valenciennes, 1840 (Oteichthyes, Characidae) causadas por Neocucullanus neocucullanus Travassos, Artigas \& Pereira 1828 (Nematoda). Acta Scientiarum 2:455-459.

Statsoft. 2003. STATISTICA for windows (computer program manual). Version 6.0. Tulsa: Statsoft, Inc.

Sverlis, S. B. \& Espinach Ros, A. 1986. El dorado, Salminus maxillosus (Pisces, Caraciformes) en el río de La Plata y río Uruguay inferior. Revista de Investigación de Desarrollo Pesquero 6:57-75.

ThÉ, A. P. G. \& Nordi, N. 2000. "O que o peixe come" na interpretação de pescadores artesanais. Revista Brasileira de Ecologia 1:55-60.

Vazzoler, A. E. A. M. 1989. Aspectos biológicos de peixes amazônicos. XI. Reprodução das espécies do gênero Semaprochilodus (Characiformes, Prochilodontidae) no baixo rio Negro, Amazonas, Brasil. Revista Brasileira de Biologia 1:165-173

1996. Biologia da reprodução de peixes teleósteos: teoria e prática. Maringá, EDUEM. $169 \mathrm{p}$.

Vazzoler, A. E. A. M. \& Menezes, N. A. 1992. Síntese de conhecimentos sobre o comportamento reprodutivo dos Characiformes da América do Sul (Teleostei, Ostariophysi). Revista Brasileira de Biologia 4:627-640.

VIEIRA, F. \& Pompeu, P. S. 2001. Peixamentos: uma alternativa eficiente? Ciência Hoje 30:28-33.

Vismara, M. R.; Benedito-Cecilio, E. \& Faria, A. C. A. 2004. Efeito da maturação gonadal sobre o conteúdo calórico e condição geral de peixes da planície de inundação do alto rio Paraná. Acta Scientiarum 2:189-199. 